\title{
Insufflation with carbon dioxide reduces pneumoperitoneum after percutaneous endoscopic gastrostomy (PEG): a randomized controlled trial
}

Authors

Institutions
Christopher J. Murphy ${ }^{1}$, Douglas G. Adler ${ }^{1}$, Kristen Cox ${ }^{1}$, Daniel N. Sommers ${ }^{2}$, John C. Fang ${ }^{1}$

${ }^{1}$ Division of Gastroenterology, Hepatology, and Nutrition, Department of Internal Medicine, University of Utah School of Medicine, Salt Lake City, Utah, United States

${ }^{2}$ Department of Radiology, University of Utah School of Medicine, Salt Lake City, Utah, United States

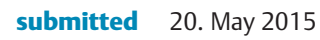
accepted after revision 16. December 2015

\section{Bibliography}

Dol http://dx.doi.org/

10.1055/s-0042-100192

Published online: 10.2.2016

Endoscopy International Open 2016; 04: E292-E295

(c) Georg Thieme Verlag KG

Stuttgart · New York

E-ISSN 2196-9736

\section{Corresponding author}

John C. Fang, MD

Division of Gastroenterology,

Hepatology, and Nutrition

Department of Internal

Medicine

The University of Utah School of Medicine

$30 \mathrm{~N} 1900 \mathrm{E}$

Room 4R118

Salt Lake City

Utah 84132

John.Fang@hsc.utah.edu
Background and study aims: Pneumoperitoneum following PEG placement has been reported in up to $60 \%$ of cases, and while usually benign and self-limited, it can lead to evaluation for suspected perforation. This study was designed to determine whether using $\mathrm{CO}_{2}$ compared to ambient air for insufflation during PEG reduces post-procedure pneumoperitoneum.

Patients and Methods: Prospective, double-blind, randomized trial of 35 consecutive patients undergoing PEG at a single academic medical center. Patients were randomized to insufflation with $\mathrm{CO}_{2}$ or ambient air. The primary outcome was pneumoperitoneum determined by left-lateral decubitus abdominal x-rays 30 minutes after PEG placement. Secondary endpoints included abdominal distention, pain, and bloating.

\section{Introduction}

$\nabla$

First described in 1980, [1] percutaneous endoscopic gastrostomy (PEG) is the most common minimally invasive method for establishing longterm enteral feeding access. The procedure is highly successful with a low overall rate of serious complications [2]. Pneumoperitoneum is relatively common after PEG, reported in $8.6 \%$ to $56 \%$ of cases, and usually self-limited, however, it may be associated with post-procedural bloating, discomfort, and pain [3-7]. Air is thought to leak into the peritoneum from full insufflation of the stomach during the between trochar needle insertion and final positioning of the gastrostomy tube [6]. In the absence of other clinical evidence of peritonitis, conservative management for postPEG pneumoperitoneum is recommended. However pneumoperitoneum can also be a sign of perforation and can lead to imaging, hospitalization, and even exploratory laparotomy after PEG placement [8].
Results: PEG was successfully placed in 17 patients using $\mathrm{CO}_{2}$ and 18 patients using ambient air. Three patients in each arm were unable or declined to have $\mathrm{x}$-rays completed and were excluded. Pneumoperitoneum was identified in 2/14 (14.3\%) using $\mathrm{CO}_{2}$ and 8/15 (53.3\%) using ambient air $(P=0.05)$. There was no significant difference in abdominal distention, visual analog scale (VAS) scores for pain or bloating between $\mathrm{CO}_{2}$ and ambient air.

Conclusion: Utilizing $\mathrm{CO}_{2}$ significantly reduces the frequency of post-procedural pneumoperitoneum compared to use of ambient air during PEG placement, with no difference in waist circumference, pain or bloating between $\mathrm{CO}_{2}$ and ambient air. $\mathrm{CO}_{2}$ appears to be safe and effective for use and may be the insufflation agent of choice during PEG.

Until recently, ambient air was the default insufflation agent for endoscopy given its ease of use, wide availability, and lack of additional costs. However, carbon dioxide is increasingly being used for both routine and therapeutic endoscopic procedures, including colonoscopy and ERCP, with consistent evidence for reduced post-procedural bloating and pain due to more rapid reabsorption [9-12]. In addition, if perforation occurs, capnoperitoneum is less pronounced and clinically detrimental due to the rapid reabsorption and its use is recommended for therapeutic endoscopic procedures [13].

The only previous study evaluating the role of $\mathrm{CO}_{2}$ insufflation during PEG placement used placement of multiple gastropexies prior to standard pull-through technique [7]. In that study, $\mathrm{CO}_{2}$ compared to ambient air was associated with less frequent post-procedural pneumoperitoneum and a reduction in small bowel distention, but pain and discomfort were not evaluated. The aim of our study was to compare $\mathrm{CO}_{2}$ vs. ambient air insufflation during PEG placement without 
the use of gastropexy for differences in the rate of post-procedural pneumoperitoneum as well as abdominal distention, pain, and bloating.

\section{Patients and methods \\ $\nabla$}

This was a single-center, randomized, double-blind prospective study conducted at the University of Utah Hospital. The study was approved by the Institutional Review Board and informed consent was obtained from patients or their surrogate medical decision-makers. Consecutive patients undergoing PEG were asked to participate from June 2012 to June 2014. Once enrolled, patients were randomized to $\mathrm{CO}_{2}$ or ambient air using a computerized random number generator. The study coordinator (KC) instructed endoscopy technicians on which insufflation agent should be used. Endoscopists and radiologists were not present for this step and remained blinded to the agent being used.

For patients in whom $\mathrm{CO}_{2}$ insufflation was used, it was delivered with the $\mathrm{CO}_{2}$ Efficient Endoscopic Insufflator (Bracco Diagnostics, Monroe Township, NJ) on the managed flow setting at $3.4 \mathrm{~L} / \mathrm{min}$. For ambient air, an Evis Exera III CLV-190 was used on the medium air flow setting (0.68 L/min). A 20F PEG (EndoVive Safety Kit, Boston Scientific, Natick, MA) was placed using the Ponsky pull method [1]. As per our standard protocol, gastropexy was not performed and the PEG tube Y-port adapter was temporarily opened immediately after placement to decompress the stomach. Procedure time, sedation doses, and any immediate complications were recorded from each procedure. No local anesthetic was used during any procedures, and there was no additional post-procedural sedation administered prior to evaluation for post-placement pain/bloating.

Post-PEG pneumoperitoneum was evaluated with a left lateral decubitus abdominal x-ray obtained within 30 minutes of endoscopic placement. All images were interpreted by an experienced gastrointestinal radiologist (DS) who was blinded to the insufflation agent used. When present, the degree of pneumoperitoneum was subjectively graded as small, moderate or large. As a surrogate for distention, abdominal circumference was measured at the level of the umbilicus with the patient supine immediately prior to PEG placement and then again within 30 minutes of the procedure. After the procedure, patients were asked to complete two visual analog scales (VAS), one for general post-procedural pain and another specifically assessing abdominal bloating. Each patient's chart was reviewed 30 days after the procedure to evaluate for any delayed complications.

Statistical analysis was performed using Stata version 10 (StataCorp LP, College Station, TX). A Fisher's exact test was used to evaluate for a significant difference in the rate of pneumoperitoneum between groups, while Student's $t$-test was used to evaluate for difference in abdominal girth and for differences in VAS scores for pain and bloating. A-priori statistical power analysis was completed and, assuming post-PEG pneumoperitoneum rates of $30 \%$ for ambient air and $10 \%$ for the $\mathrm{CO}_{2}$ group, we calculated that 15 patients were needed in each arm to reach the standard statistical power threshold of 0.8 . A $P$ value $\leq 0.5$ was considered statistically significant.

\section{Results}

$\nabla$

A total of 35 subjects undergoing PEG placement were enrolled during the study period, of whom 17 patients were randomized to $\mathrm{CO}_{2}$ and 18 patients were randomized to ambient air. Indications for placement included amyotrophic lateral sclerosis (ALS, $\mathrm{N}=18$ ), neurogenic dysphagia $(\mathrm{N}=13)$, malnutrition ( $>10 \%$ loss of ideal body weight) $(\mathrm{N}=1)$, and high aspiration risk $(\mathrm{N}=3)$. The preponderance of patients with ALS is because the University of Utah is the regional referral center for the disease.

Patient demographics and procedural indications are summarized in Table 1 . There were no statistically significant differences between groups, although there was a trend toward more females with ALS in the ambient air group. Placement was successful in all cases without any immediate complications. Tube feedings were initiated uneventfully and reached goal rates without unexpected delays. There were no subsequent hospitalizations or reported complications within 30 days of PEG placement for any patient.

Post-procedural left lateral decubitus x-rays were obtained within 30 minutes of PEG placement except in three patients in the $\mathrm{CO}_{2}$ group and three patients in the ambient air group, leaving 14 patients randomized to $\mathrm{CO}_{2}$ and 15 patients to ambient air. One patient had an error in order entry that led to prolonged delay in imaging, and the other five patients declined to participate in additional imaging due to postoperative discomfort or excessive sedation within the 30-minute window.

Outcomes of PEG placement using $\mathrm{CO}_{2}$ vs ambient air are summarized in Table 2. Pneumoperitoneum was significantly less frequent when using $\mathrm{CO}_{2}$, with post-procedure pneumoperitoneum in $2 / 14(14.3 \%)$ compared to $8 / 15$ (53.3\%) using ambient air $(P=0.05)$. There was no significant difference in the radiographic size of pneumoperitoneum between groups $(P=0.65$, - Table2). There was no significant difference in pre- and postprocedure abdominal circumference, with a mean change in waist measurement of $3.1 \pm 3.1 \mathrm{~cm}$ in the $\mathrm{CO}_{2}$ group vs. $3.0 \pm$ $2.5 \mathrm{~cm}$ in the ambient air group $(P=0.85)$.

Patients with end-stage ALS often had difficulty interacting with the VAS, especially after procedural sedation, and there was a high dropout in this aspect of the study, limiting its value and statistical power. Six patients in the $\mathrm{CO}_{2}$ arm (3 with ALS) and nine patients in the ambient air arm (4 with ALS) declined or were unable to complete the VAS. Only 11 patients in the $\mathrm{CO}_{2}$ and nine patients in the ambient air group completed VAS. There were no significant differences in scores for abdominal pain (4.3 vs. $2.2, P=0.12$ ) or for abdominal bloating ( 2.8 vs. $1.9, P=0.46$ ).

\section{Discussion \\ $\nabla$}

This study showed a significant decrease in the frequency of postprocedural pneumoperitoneum when using $\mathrm{CO}_{2}$. We report a rate of post-PEG pneumoperitoneum of $53 \%$ when using ambient air compared to only $14.3 \%$ with $\mathrm{CO}_{2}$. This is the first study to measure and evaluate subjective measures of pain and bloating after PEG using $\mathrm{CO}_{2}$ insufflation, however, it did not find a significant difference in abdominal girth or in patient-reported pain or bloating between $\mathrm{CO}_{2}$ and ambient air insufflation.

Incidence of post-PEG pneumoperitoneum using ambient air varies widely in published reports (8.6\%-56\%) [4-7]. This is likely due to numerous differences in study design, including timing and sensitivity of imaging modalities and differences in gastros- 
Table 1 Demographics of patients undergoing PEG using $\mathrm{CO}_{2}$ vs. ambient air for insufflation.

\begin{tabular}{|c|c|c|c|}
\hline & $\mathrm{CO}_{2}$ & Ambient Air & $P$ value \\
\hline No. of patients & 17 & 18 & \\
\hline Age $($ Mean $\pm S D)$ & $55.3 \pm 22.1$ & $55.6 \pm 13.6$ & 0.87 \\
\hline $\operatorname{Sex}(N, \%)$ & & & 0.09 \\
\hline Males & $10(58 \%)$ & $5(28 \%)$ & \\
\hline Females & $7(41 \%)$ & $13(72 \%)$ & \\
\hline BMI (Mean \pm SD) & $20.7 \pm 5.2$ & $22.5 \pm 6.6$ & 0.39 \\
\hline \multicolumn{4}{|l|}{ Indication (N,\%) } \\
\hline ALS & $6(35.3 \%)$ & $12(66.7 \%)$ & 0.11 \\
\hline Neurogenic dysphagia & $7(41.2 \%)$ & $6(33.3 \%)$ & \\
\hline Malnutrition & $1(5.9 \%)$ & 0 & \\
\hline High aspiration risk & $3(17.9 \%)$ & 0 & \\
\hline
\end{tabular}

PEG, percutaneous endoscopic gastrostomy; BMI, body mass index; ALS, amyotrophic lateral sclerosis

Table 2 Outcomes of PEG placement using $\mathrm{CO}_{2}$ vs. ambient air for insufflation.

\begin{tabular}{|c|c|c|c|}
\hline & $\mathrm{CO}_{2}$ & Ambient Air & $P$ value \\
\hline Pneumoperitoneum (N,\%) & & & 0.05 \\
\hline Free Air & $2(14.3 \%)$ & $8(53.3 \%)$ & \\
\hline No Free Air & $12(85.7 \%)$ & $7(46.7 \%)$ & \\
\hline Pneumoperitoneum grade (N) & & & 0.65 \\
\hline Small & 1 & 4 & \\
\hline Moderate & 1 & 2 & \\
\hline Large & 0 & 2 & \\
\hline \multicolumn{4}{|l|}{ Abdominal girth } \\
\hline $\begin{array}{l}\text { Change in circumference } \\
(\mathrm{cm}) \text { Mean } \pm \text { SD }\end{array}$ & $3.1 \pm 3.1 \mathrm{~cm}$ & $3.3 \pm 2.5 \mathrm{~cm}$ & 0.85 \\
\hline Pain VAS (mean \pm SD) & $4.3 \pm 3.0$ & $2.2 \pm 2.4$ & 0.12 \\
\hline Bloating VAS (mean \pm SD) & $2.8 \pm 3.0$ & $1.9 \pm 2.0$ & 0.46 \\
\hline \multicolumn{4}{|l|}{ Procedure duration and sedation } \\
\hline Mean procedure time (mins) & $13.0 \pm 10.5$ & $12.5 \pm 8.2$ & 0.88 \\
\hline Fentanyl $(\mathrm{mcg})$ mean \pm SD & $43 \pm 32$ & $47 \pm 19$ & 0.66 \\
\hline Propofol $(\mathrm{mg})$ mean \pm SD & $175 \pm 119$ & $163 \pm 54$ & 0.69 \\
\hline
\end{tabular}

PEG, percutaneous endoscopic gastrostomy; VAS, visual analog scale

tomy technique. The lowest reported rate $(8.6 \%)$ was a retrospective study of standard chest X-rays not specifically obtained to evaluate for post-PEG pneumoperitoneum. Other studies obtaining plain upright and AP chest X-rays (rate $20.9 \%$ ) or plain abdominal films obtained in a variety of positions (rate $38 \%$ ) performed within 3 hours of PEG reported intermediate rates of pneumoperitoneum $[4,6]$. Abdominal computed tomography $(\mathrm{CT})$, the most sensitive imaging modality for identifying intraperitoneal free air, was used in two studies. One series obtaining CT 24 hours after PEG reported a rate of $26.7 \%$, however, as previously noted, this study used gastropexy [7]. In the second series, in which abdominal CT scans were obtained from 1 hour to 9 days after gastrostomy, the rate was $56 \%$, but this study was performed by Interventional Radiology under push-technique with dilators, which likely increased the incidence of pneumoperitoneum [5]. Our method of using a fairly sensitive technique, left lateral decubitus film, obtained soon (within 30 minutes) after PEG placement likely resulted in our incidence of pneumoperitoneum air being at the higher end of the reported range. Our study is the second report to evaluate $\mathrm{CO}_{2}$ insufflation during PEG and confirms previous results demonstrating that $\mathrm{CO}_{2}$ use significantly reduces the rate of post-PEG pneumoperitoneum [7]. Our rate of capnoperitoneum was $14.3 \%$, substantially higher than the previous study of 58 patients, which reported a rate of $0 \%$. Our higher rate of capnoperitoneum may be explained by two factors. First, our left lateral decubitus X-rays were obtained 30 minutes after placement, compared to their CT scans, which were obtained 24 hours after placement. It is very likely that relatively small amounts of $\mathrm{CO}_{2}$ within the peritoneum will be completely resorbed within 24 hours; therefore, our timelier imaging likely gives a more accurate rate of the immediate post-procedural capnoperitoneum rate. Second, the previous study used three gastropexies during PEG placement prior to trocar insertion and pull PEG placement. We found higher rates of pneumoperitoneum when either ambient air (53\% vs. $27 \%$ ) or $\mathrm{CO}_{2}(14 \%$ vs. $0 \%)$ was used, suggesting that gastropexy reduces air leakage into the peritoneum during PEG regardless of insufflation agent. Gastropexy is common during PEG placement in Japan, but is not widely used in the United States, making our results more relevant when gastropexies are not used.

We found no difference between ambient air and $\mathrm{CO}_{2}$ in change of abdominal girth before and after the procedure. Previous studies of upper and lower endoscopy using similar methodologies for measuring abdominal girth did report differences in abdominal girth when insufflating with $\mathrm{CO}_{2}$ vs. ambient air $[11,14]$. Our patients were vented immediately after PEG placement, which may have contributed to the lack of difference in abdominal girth between groups. In addition, PEG procedures are significantly shorter than ERCP and colonoscopy with less volume of insufflation agent, thus this method may not be sensitive enough to detect relatively smaller differences in intraluminal bowel distention. In the previously cited study evaluating $\mathrm{CO}_{2}$ vs. ambient air for PEG, KUB was used to measure bowel distention after PEG and less distension in the small bowel was reported with $\mathrm{CO}_{2}$ but no difference in distention was reported in the colon [7]. Our method of abdominal girth measurement may not be sensitive enough to have detected differences in distention isolated to the small bowel.

This is the first study to evaluate for differences in patient-reported pain and bloating between $\mathrm{CO}_{2}$ and ambient air during PEG placement. We found no difference in VAS scores in pain or bloating, although several patients were unable to participate in the evaluation shortly after placement due to severe neurologic impairment (end-stage ALS) or persistent sedation. Other endoscopic procedures, including colonoscopy and endoscopic retrograde cholangiopancreatography (ERCP), have been associated with significant improvement in post-procedural bloating and pain when using $\mathrm{CO}_{2}$. The fact that PEG placement did not seem to show a benefit in symptoms may be explained by the shorter duration of PEG and the lower total volume of insufflation introduced, as well as the fact that patients undergoing PEG have more comorbidities and may be less prone to notice minor differences in symptoms.

Our study also confirms that post-PEG pneumoperitoneum by itself is a clinically benign finding. We agree that in the absence of other clinical signs of perforation (e.g. peritoneal signs, subcutaneous emphysema, or sepsis), post-PEG pneumoperitoneum does not require intervention or warrant further workup. However, the lower incidence and more rapid resolution of benign pneumoperitoneum with $\mathrm{CO}_{2}$ may help prevent confusion regarding the need for additional imaging, evaluation, and even laparotomy to rule out perforation.

This study has several important limitations. It enrolled fewer patients than previous series, and not all of them participated in the post-procedure $\mathrm{x}$-ray to evaluate for pneumoperitoneum. How- 
ever, equal numbers of patients were lost in each group and we did reach our predetermined enrollment size for the primary outcome as calculated by a-priori power analysis. Not all patients were willing or physically capable of participating in VAS quantification of post-procedural pain and bloating. This aspect of the study was limited by the smaller numbers of patients participating in VAS scoring, however, there was no dramatic difference in VAS scores. A slight difference in symptoms between $\mathrm{CO}_{2}$ and ambient air is unlikely to have clinical relevance, given that PEG patients are often fairly debilitated with high rates of comorbid disease. Plain $\mathrm{X}$-ray is a less sensitive modality than abdominal CT for demonstrating pneumoperitoneum. We compensated, however, by obtaining images within 30 minutes of PEG placement, using left lateral decubitus films, and having a single highly experienced gastrointestinal radiologist interpret all the films. Measurement of abdominal girth at the level of the umbilicus may not be sensitive or reliable enough to detect smaller differences in bowel distention or pneumoperitoneum, however, we felt that obtaining abdominal CTs in patients without other clinical signs or symptoms was not clinically indicated and was prohibitively expensive. Ambient air insufflation produces noise compared to the relatively silent pressurized $\mathrm{CO}_{2}$ system, which may have affected blinding of the endoscopist during PEG placement. However, we believe this effect was minimal because our suction vacuum produces significantly more noise than other insufflation systems, and the radiologist and assistant measuring study variables were not present during actual endoscopy.

\section{Conclusions}

\section{$\nabla$}

$\mathrm{CO}_{2}$ significantly reduces the frequency of post-procedural pneumoperitoneum compared to ambient air as an insufflation agent during PEG placement. There were no significant differences in measured abdominal distension or patient-reported symptoms of abdominal bloating or pain. Reduction in the incidence and duration of pneumoperitoneum may help prevent benign pneumoperitoneum being misconstrued as a sign of perforation, preventing unnecessary evaluation, imaging, and even surgical intervention. Carbon dioxide appears to be safe and effective and may be the insufflation agent of choice for PEG placement.

\section{Competing interests: None}

\section{Acknowledgement}

$\nabla$

This study was approved by the University of Utah Institutional Review Board and all patients completed informed consent prior to enrollment.

\section{References}

1 Gauderer MW, Ponsky JL, Izant RJ. Gastrostomy without laparotomy: a percutaneous endoscopic technique. J Ped Surg 1980; 15: 872 - 875

2 Schrag SP, Sharma R, Jaik NP et al. Complications related to percutaneous endoscopic gastrostomy (PEG) tubes. A comprehensive clinical review. J Gastrointestin Liver Dis 2007; 16: 407-418

3 Dulabon GR, Abrams JE, Rutherford EJ. The incidence and significance of free air after percutaneous endoscopic gastrostomy. Am Surg 2002; 68: $590-593$

4 Wiesen AJ, Sideridis K, Fernandes A et al. True incidence and clinical significance of pneumoperitoneum after PEG placement: a prospective study. Gastrointest Endosc 2006; 64: 886-889

5 Wojtowycz MM, Arata JA Jr., Micklos TJ et al. CT findings after uncomplicated percutaneous gastrostomy. AJR Am J Roentgenol 1988; 151: 307-309

6 Gottfried EB, Plumser AB, Clair MR. Pneumoperitoneum following percutaneous endoscopic gastrostomy. A prospective study. Gastrointest Endosc 1986; 32: 397-399

7 Nishiwaki S, Araki H, Hayashi M et al. Inhibitory effects of carbon dioxide insufflation on pneumoperitoneum and bowel distension after percutaneous endoscopic gastrostomy. World J Gastroenterol 2012; 18: 3565-3570 (DOI 10.3748/wjg.v18.i27.3565)

8 Snape J, Poon M, Green J et al. Pneumoperitoneum following PEG insertion: a clinical dilemma. Acute Med 2010; 9: 120-121

$9 \mathrm{Wu}, \mathrm{Hu} \mathrm{B}$. The role of carbon dioxide insufflation in colonoscopy: a systematic review and meta-analysis. Endoscopy 2012; 44: 128-136 (DOI 10.1055/s-0031-1291487)

10 Bretthauer M, Seip B, Aasen S et al. Carbon dioxide insufflation for more comfortable endoscopic retrograde cholangiopancreatography: a randomized, controlled, double-blind trial. Endoscopy 2007; 39: 58-64

11 Maple JT, Keswani RN, Hovis RM et al. Carbon dioxide insufflation during ERCP for reduction of postprocedure pain: a randomized, doubleblind, controlled trial. Gastrointest Endosc 2009; 70: 278-283 (DOI 10.1016/j.gie.2008.12.050)

12 Dellon ES, Hawk JS, Grimm IS et al. The use of carbon dioxide for insufflation during GI endoscopy: a systematic review. Gastrointest Endosc 2009; 69: 843-849 (DOI 10.1016/j.gie.2008.05.067)

13 Paspatis GA, Dumonceau JM, Barthet $M$ et al. Diagnosis and management of iatrogenic endoscopic perforations: European Society of Gastrointestinal Endoscopy (ESGE) Position Statement. Endoscopy 2014; 46: $693-711$

14 Seo EH, Kim TO, Park MJ et al. The efficacy and safety of carbon dioxide insufflation during colonoscopy with consecutive esophagogastroduodenoscopy in moderately sedated outpatients: a randomized, double-blind, controlled trial. J Clin Gastroenterol 2013; 47: 45-49 